\title{
Cinco especies nuevas de Pselliopus (Hemiptera: Heteroptera: Reduviidae: Harpactorinae: Harpactorini) para México
}

\author{
Five new species of Pselliopus (Hemiptera: Heteroptera: Reduviidae: Harpactorinae) from \\ Mexico
}

\author{
Harry Brailovsky*, Ricardo Mariño y Ernesto Barrera \\ Departamento de Zoología, Instituto de Biología, Universidad Nacional Autónoma de México. Apartado postal 70153, 04510 México D.F. México. \\ *Correspondencia: coreidae@servidor.unam.mx
}

\begin{abstract}
Resumen. Se describen 5 especies nuevas de Pselliopus Bergroth (Reduviidae: Harpactorinae) recolectadas en México (Guerrero, Hidalgo, Jalisco, Michoacán, Nayarit, Nuevo León, Oaxaca, Puebla, Querétaro, San Luis Potosí, Sinaloa, Sonora y Tamaulipas) y en los Estados Unidos de América (Arizona). Pselliopus karlenae Hussey se registra por primera vez para la República Mexicana. Se ilustran caracteres diagnósticos del pronoto, del borde posterior del segmento abdominal VII, de la cápsula genital del macho (pigóforo) y parámeros. Se incluye una clave para la identificación de las especies basada principalmente en los genitales masculinos.
\end{abstract}

Palabras clave: Insecta, Hemiptera, Reduviidae, Harpactorinae, Pselliopus, especies nuevas, clave, México.

\begin{abstract}
Five new species of Pselliopus Bergroth (Reduviidae: Harpactorinae) from Mexico (Guerrero, Hidalgo, Jalisco, Michoacán, Nayarit, Nuevo León, Oaxaca, Puebla, Querétaro, San Luis Potosí, Sinaloa, Sonora and Tamaulipas) and from the United States of America (Arizona) are described. Pselliopus karlenae Hussey is a new record for Mexico. Diagnostic taxonomic characters of pronotum, posterior border of abdominal segment VII, male genital capsule (pygophore) and parameres are illustrated. An identification key based mainly on the male genitalia is included.
\end{abstract}

Key words: Insecta, Hemiptera, Reduviidae, Harpactorinae, Pselliopus, new species, key, Mexico.

\section{Introducción}

La subfamilia Harpactorinae, de acuerdo con los datos proporcionados por Maldonado Capriles (1990), está constituida por 2059 especies en 288 géneros, en tanto que Schuh y Slater (1995) reconocen 2000 especies en 278 géneros en el mundo. En México se registran 20 géneros con 81 especies (Coscarón, 2002) y habitualmente se les encuentra en vegetación baja, en hojarasca o árboles caídos y durante el día en lugares soleados, siendo sumamente activos.

La tribu Harpactorini incluye 19 de los 20 géneros de la subfamilia Harpactorinae, con 64 especies registradas para México. De los 19 géneros citados para México ninguno se encuentra en el Viejo Mundo, y comparte todos con América del Sur con excepción de Fitchia Stål (monoespecífico) (Coscarón, 2002).

Pselliopus Bergroth es exclusivamente americano, incluye

Recibido: 07 agosto 2006; aceptado: 11 octubre 2006
22 especies, de las cuales 15 se distribuyen en México ( $P$. dantei Brailovsky y Barrera, P. flaviceps Brailovsky y Barrera, $P$. inermis [Champion], $P$. ivanicus Brailovsky y Barrera, P. latispina Hussey, P. lineaticeps [Champion], $P$. majesticus Brailovsky y Barrera, P. mexicanus [Champion], P. nigropictus [Champion], P. punctipes (Amyot y Serville), $P$. rufofasciatus [Champion], $P$. spinicollis [Champion], $P$. tuberculatus [Champion], P. ventus Brailovsky y Barrera y $P$. zebra [Stål]); una está citada para Canadá ( $P$. cinctus [Fabricius]), 3 para los Estados Unidos de América ( $P$. barberi Davis, P. cinctus [Fabricius] y P. latifasciatus Barber), una de Guatemala (P. infuscatus [Champion]), una de Honduras (P. karlenae Hussey), y 2 de Argentina y Brasil (P. ornaticeps [Stål]) y P. limai Pinto) (Stål 1862, 1872, Champion, 1899; Barber, 1924; Brailovsky y Barrera, 2004; Fracker, 1912; Hussey, 1954; Maldonado Capriles, 1990; Coscarón, 2002).

De las especies que se encuentran en México, $P$. inermis y $P$. spinicollis se citan también para los Estados Unidos de América, $P$. punctipes para El Salvador y Surinam y $P$. 
zebra se distribuye desde los Estados Unidos de América hasta Panamá.

Las especies de este género depredan insectos y otros artrópodos terrestres, mostrando una coloración llamativa rojiza y amarillenta, caracterizándose por tener en el hemélitro una areola cuadrangular, el artejo rostral I más corto que el II y el fémur anterior más corto que el posterior (Fracker, 1912).

Como resultado de este estudio se describen como nuevas 5 especies y una ( $P$. karlenae) queda como nuevo registro para México, por lo que el número de especies se eleva a 27, de las cuales 21 se encuentran en la República Mexicana (Brailovsky y Barrera 2004).

Acrónimos usados en el texto. BMNH (The Natural History Museum, Londres); CAS (California Academy of Sciences, San Francisco, California); CMNH (Carnegie Museum of Natural History; Pittsburgh, Pennsylvania); IBUNAM (Colección Entomológica, Instituto de Biología, Universidad Nacional Autónoma de México), y UCB (Universidad de California, Berkeley, California).

Rasgos comunes para las especies nuevas. Las especies descritas comparten algunos caracteres estructurales y de coloración que se incluyen en este apartado para no ser reiteradamente mencionados y sólo resaltar los caracteres relevantes de cada taxón.

Coloración. Antena. Artejo antenal I negro con cuatro anillos amarillos; artejo antenal II negro con un anillo amarillo y los artejos III y IV pardo claro a pardo castaño anaranjado. Rostro. Artejo rostral I amarillo con la unión basal y 2 manchas cercanamente cuadradas hacia el tercio medio de color negro; artejo rostral II amarillo con la unión basal negra y el III negro o pardo oscuro. Escutelo. Tercio anterior negro con 2 manchas tomentosas blanquecinas y el resto, incluyendo el ápice, amarillo. Membrana hemelitral. Amarillo oscuro y con las venas más oscuras. Conexivo. Segmentos conexivales del abdomen amarillos y con el tercio anterior negro (borde anterior amarillo). Tórax. Acetábulos de los 3 pares de patas amarillos. Patas. Trocánteres amarillos y con el borde externo y la espina negra; tarsos basal y medio pardo claro o anaranjado oscuro y el apical pardo oscuro. Abdomen. Margen pleural II amarillo y del III al VII amarillos y con el tercio anterior negro (borde anterior amarillo). Estructuras. Cabeza. Más corta que la longitud total del pronoto. Pronoto. Trapeziforme, bilobulado; lóbulo anterior más corto que el posterior; ángulos frontales dirigidos hacia fuera en un diente cónico, ancho y casi romo; lóbulo anterior liso con el tercio medio profundamente surcado, el cual se continúa hacia el lóbulo posterior donde el surco se ensancha; lóbulo posterior finamente punteado; márgenes posterolaterales algo sinuados; procesos triangulares redondeados; borde posterior algo sinuado. Escutelo. Ápice redondeado y conspicuamente foliado. En las descripciones las medidas se expresan en milímetros.

Las hembras no presentan caracteres estructurales o de coloración suficientemente conspicuos para ser utilizados en el reconocimiento de especie, por lo cual sólo cuando ambos sexos fueron recolectados al unísono se pudo inferir el nivel especifico de la hembra y su asociación al macho correspondiente.

\section{Descripción}

Pselliopus cosmopolites n. sp. (Figs. 9-10, 31, 37, 44)

Holotipo macho. Coloración dorsal. Cabeza. Negra, con los juga, una mancha triangular entre los tubérculos anteníferos, una mancha discoidal interocelar y la zona adyacente a los ojos de color amarillo. Pronoto. Lóbulo anterior amarillo con las siguientes áreas de color negro: cara interna de los ángulos frontales, 2 manchas longitudinales que corren a lo largo de la línea media, una franja transversal que lo separa del lóbulo posterior, 2 franjas oblicuas laterales a la línea media, y una mancha ramificada en el tercio anterior de los márgenes anterolaterales y colocadas por debajo de los ángulos frontales; lóbulo posterior amarillo o rojizo, con las espinas humerales negras y los bordes posterolaterales (tercio medio con una mancha discoidal parda o negra), los procesos triangulares y el margen posterior de color crema. Hemélitro. Clavus y corium pardo claro. Abdomen. Segmentos dorsales negros y con el margen posterior del VII amarillo. Coloración ventral. Cabeza. Amarilla y con una franja longitudinal negra que corre de la zona postocular al cuello. Tórax. Protórax amarillo con una mancha negra en "H" cubriendo parte de la propleura; mesotórax y metatórax amarillos y densamente pilosos; mesopleura amarilla con una mancha negra y ancha en zigzag; metapleura amarilla con una mancha negra en "c" invertida. Patas. Coxas amarillas con la cara interna mayormente negra; fémures amarillos con 5 anillos negros (el primero incompleto) y un punto también negro entre los anillos 3 y 4; tibia anterior amarilla con 5 anillos y la espina apical negros; tibia media amarilla y con 5 anillos negros; tibia posterior amarilla con 4 anillos negros y una mancha alargada parda o negra en la cara externa de la segunda mitad del artejo. Abdomen. Esternitos amarillos con una franja transversal negra en el margen posterior, y que en los esternitos II al VI lateralmente y cercana al margen pleural se ensancha, dando el aspecto de un círculo irregular negro; pigóforo amarillo con franjas negras laterales a la línea media que se unen en la parte basal del borde posteroventral; parámeros negros y con la base amarilla. Estructura. Pronoto. Ángulos humerales 
proyectados en una espina corta, robusta y dirigida hacia fuera (Fig. 37). Abdomen. Borde posterior del segmento VII suavemente convexo (Fig. 31). Pigóforo. Borde posteroventral proyectado en su tercio medio en una saliente mediana, apicalmente bífida que casi toca el borde posterior del segmento abdominal VII y con la base algo ensanchada; lóbulos genitales redondeados (Fig. 9); parámeros robustos, largos, curvos, terminados en forma de mazo, clavados, con el ápice piloso y rebasando el bode posterior del segmento abdominal VII. (Fig. 10).

Hembra. Coloración semejante al holotipo macho. Placas genitales amarillas con bandas oblicuas de color negro en el tercio apical.

Variación. La zona amarilla adyacente a los ojos penetra hacia el espacio interocular, llegando casi a unirse con la mancha del lado opuesto. Lóbulo anterior del pronoto negro con el margen anterior, una mancha longitudinal irregular a cada lado de la línea media, los márgenes anterolaterales y una pequeña mancha cercana al lóbulo posterior de color amarillo. Lóbulo posterior del pronoto de color crema, rojo, o anaranjado, con las espinas humerales negras y los márgenes posterolaterales (excepto el tercio medio que es negro) y el margen posterior amarillo. Márgenes posterolaterales del pronoto enteramente amarillos o crema. Manchas negras de la propleura, mesopleura y metapleura desvanecidas.

Medidas. Primero el macho después la hembra. Cabeza. Longitud total: 2.28, 2.28; anchura a través de los ojos: 1.20, 1.20; distancia interocular: 0.60, 0.72; longitud total de los artejos antenales: I; 3.60, 3.60; II, 1.56, 1.44; III, 2.76, 2.40; IV, 1.56, 1.32. Pronoto. Longitud total del lóbulo anterior: 1.08, 0.84; longitud total del lóbulo posterior: 1.68, 1.68; anchura máxima del lóbulo anterior: 1.80, 1.80; anchura máxima del lóbulo posterior: 3.36, 3.36. Longitud total del cuerpo: 11.52, 11.88.

\section{Resumen taxonómico}

Holotipo: macho. MÉXICO. QUERÉTARO. Km 8 carr., Jalpan-Landa de Matamoros, 1170 m, 21 ${ }^{\circ} 19^{\prime}$ N-99 $51^{\circ}$ O', 18-X-1997, E. Barrera y H. Brailovsky (IBUNAM). Paratipos. MÉXICO. QUERÉTARO. 2 machos, 1 hembra, km 8 carr. Jalpan-Landa de Matamoros, $1170 \mathrm{~m}$, 2119'-N:9951'O, 18-X-1997, E. Barrera y H. Brailovsky (IBUNAM); 2 machos, 4 hembras, Pinal de Amoles, 2880 m, 2113'N-9962'O, 22-X-1995, C. A. Sánchez, 17-X1997, H. Brailovsky y E. Barrera, 25-VI-1998, G. Ortega León y L. Cervantes (IBUNAM); 1 macho, Tancoyol, 21³9’N-99³2’O, 17-XI-1991, H. Brailovsky y E. Barrera (IBUNAM); 1 hembra, Puente de Dios. Laguna de Tepisquintla, 1040 m, 21 ${ }^{\circ} 11^{\prime}$ N-99³0'O, 25-VII-1998, E. Barrera y C. Mayorga (IBUNAM); 1 hembra, km 127 carr. Vizarrón-Jalpan, 2230 m, 21 $21^{\prime}$ N-9947’ O, 17-X-
1997, H. Brailovsky y E. Barrera (IBUNAM), 1 hembra, km 5 Tilaco-Agua Zarca, 1070 m, $21^{\circ} 18^{\prime}$ N-99 $22^{\circ}$ O, 21 VII-1998, E. Barrera y C. Mayorga (IBUNAM), 1 hembra, km 8 San Joaquín de las Ranas, 2450 m, 20 55'28' 'N99³3'53' 'O, 15-X-1997, H. Brailovsky y E. Barrera (IBUNAM); 1 hembra, Cadereyta, 2069'N-9981'O, 14-XI-1991, H. Brailovsky y E. Barrera (IBUNAM); 1 hembra, Toluquilla, $2540 \mathrm{~m}, 20^{\circ} 84^{\prime} \mathrm{N}-99^{\circ} 52^{\prime} \mathrm{O}, 16-\mathrm{X}-$ 1997, H. Brailovsky y E. Barrera (IBUNAM); 1 hembra, km 4 La Lagunita-Tilaco, 1160 m, 21 ${ }^{\circ} 18^{\prime}$ N-99 $18^{\circ}$ O, 18X-1997, H. Brailovsky y E. Barrera (IBUNAM); 1 hembra, Las Ruinas Toluquilla, 2053'04' N-99³1'47' O, 2III-1998, G. Ortega y E. Barrera (IBUNAM). NUEVO LEÓN. 3 machos, 6 hembras, km 23 carr. Linares-San Roberto, 2472'N-9990'O, 22-37-IX-1983, M. García y A. Ibarra (IBUNAM); 1 macho, 7 hembras, km 30 carr. Linares-San-Roberto, 2424'N-9987' O, 18-XI-1985, F. Arias (IBUNAM); 1 macho, Santiago Las Adjuntas, 254'ㅅ-100¹5'O, 7-XI-1985, R. Barba, S. Tufinio, H. Rojas y A. Contreras (IBUNAM); 1 macho, Chipinque, $25^{\circ} 61^{\prime} \mathrm{N}-100^{\circ} 35^{\prime} \mathrm{O}, 23-\mathrm{VI}-1977$, E. Barrera (IBUNAM); 1 macho, Mpio. De Santiago El Yerbaniz, 22-XI-1986, A. Contreras (IBUNAM); 2 machos, Cola de Caballo, 253'스-100¹6’O, 22-XI-1985, F. Arias y L. Cervantes (IBUNAM). TAMAULIPAS. 1 macho, Altas Cumbres,

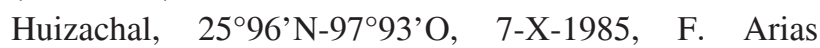
(IBUNAM); 1 macho, Cd. Victoria, 2373'N-99¹4'O, 17-XI-1977, H. Brailovsky (IBUNAM). HIDALGO. 1 macho, 1 hembra, Laguna Atezca, 22-VII-1979, R. Terrón (IBUNAM). SAN LUIS POTOSÍ. 2 machos, km 172 carr. Tasquillo-Tamazunchale, $21^{\circ} 26^{\prime} \mathrm{N}-98^{\circ} 78^{\prime} \mathrm{O}, 10-\mathrm{IV}$ 1980, H. Brailovsky (IBUNAM). PUEBLA. 1 macho, Tehuacán San Francisco de la Cañada, 1849’N-97³9' O, 13-III-1992, H. Brailovsky y E. Barrera (IBUNAM); 1 macho, Nicolás Bravo, 1861'N-97²9'O, 16-X-1992, H. Brailovsky y C. Mayorga (IBUNAM); km 12 carr. Tehuacán-Teotitlán del Camino, 1846’N-97³9'O, 29-III-1992, E. Barrera y C. Mayorga (IBUNAM); 1 hembra, Texmelucan, 19²8'N-9843'O, 1-XI-1990, H. Brailovsky y E. Barrera (IBUNAM); 2 hembras, $3 \mathrm{~km}$ al NO de Tecaltzingo, $18^{\circ} 71^{\prime}$ N-97²67' O, 13,20-VII-1992, E. Barrera y C. Mayorga (IBUNAM); 2 hembras, San Esteban Necoxcalco, $18^{\circ} 46^{\prime}$ N-97²9'O, 6-I-1993, H. Brailovsky y E. Barrera (IBUNAM); 1 hembra, Nicolás Bravo, $18^{\circ} 61^{\prime} \mathrm{N}-$ 97²9'O, 1-IX-1992, E. Barrera y G. Ortega (IBUNAM); 1 hembra, Tehuacán Sta. Ana, $18^{\circ} 37^{\prime}$ N-97 $57^{\circ}$ O, 15-VI1992, H. Brailovsky y E. Barrera (IBUNAM); 1 hembra, La Trinidad, $5 \mathrm{~km}$ al SE de Atlixco, 18 $50^{\circ} \mathrm{N}-98^{\circ} 25^{\prime} \mathrm{O}$, 15-VI-1994, E. Barrera y C. Mayorga (IBUNAM). OAXACA. 1 macho, Macuiltxóchitl, 1701'N-9653'O, 9-IX-1979, E. Mariño (IBUNAM); 1 macho, Monte Albán, 1703'N-9676'O, 16-VI-1986, M. García (IBUNAM); 1 
hembra, km 56 carr. Oaxaca-San Miguel Sola de Vega, $16^{\circ} 68^{\prime}$ N-9683'O, 1-VII-1990, E. Barrera y A. Cadena (IBUNAM).

Etimología. Nombre específico haciendo referencia a su amplia distribución; del latín, cosmopolites.

\section{Comentarios taxonómicos}

Los parámeros clavados (Fig.10) y el borde posteroventral del pigóforo proyectado en su tercio medio en una saliente mediana (Fig. 9) definen a P. cosmopolites, el cual comparte con P. mexicanus los parámeros clavados (Fig. 16); sin embargo, en este último la proyección media del borde posteroventral del pigóforo está reducida a una minúscula saliente (Fig. 15).

Pselliopus rayonensis n. sp. (Figs. 17, 18, 32, 38, 45)

Holotipo macho. Coloración dorsal. Cabeza. Amarilla con el tylus, los tubérculos anteníferos, una mancha interocular en "v", los tubérculos ocelares y una mancha que corre de la región postocular a la primera mitad del cuello (excepto por una franja amarilla que corre del espacio interocelar al cuello) de color negro; la segunda mitad del cuello anaranjada. Pronoto. Lóbulo anterior anaranjado, con el margen anterior y los ángulos frontales amarillos y con una franja discontinua pardo oscura que lo separa del lóbulo posterior; lóbulo posterior amarillo con el primer tercio del surco medio, las espinas humerales, y una mancha a cada lado de la línea media del margen posterior de color pardo o negro. Hemélitro. Tercio apical del clavus y el endocorium pardo castaño; tercio basal del clavus y el exocorium pardo claro a amarillo. Abdomen. Segmentos dorsales II a VI pardo oscuro con una franja negra en la margen anterior y el VII negro y con la mitad posterior amarilla. Coloración ventral. Cabeza. Amarilla con la parte basal del rostro y una franja que corre de la región postocular al cuello de color negro. Tórax. Protórax anaranjado con el prosterno amarillo; mesotórax con el mesosterno y el margen posterior de la metapleura amarilla, el margen anterior y cercano a la línea media anaranjada y la cara externa y anterior de la propia mesopleura negra; metatórax negro con el metasternum y el margen posterior de la metapleura amarilla; mesotórax y metatórax densamente pilosos. Patas. Coxa anterior anaranjada; coxa media amarilla y con una franja negra en la cara anterior; coxa posterior amarilla y con el borde posterior negro; fémures amarillos con 3 anillos negros y con un punto discoidal también negro situado en la cara anterior de los anillos III y IV; tibia anterior amarilla con 3 anillos y la espina basal negra; tibia media amarilla y con 4 anillos negros; tibia posterior amarilla con 3 anillos negros y una mancha subapical alargada pardo claro. Abdomen. Esternitos abdominales
II a VI con 3 manchas subcuadradas amarillas delimitadas por expansiones negras y el VII amarillo; margen posterior de cada esternito negro que lateral y cercano al margen pleural se ensancha, dando el aspecto de un círculo irregular negro; pigóforo amarillo con 2 franjas negras ensanchadas laterales a la línea media que se unen en la parte basal del borde posteroventral; parámeros negros. Estructura. Pronoto. Ángulos humerales proyectados en una espina mediana, robusta y dirigida hacia fuera (Fig. 38). Abdomen. Borde posterior del segmento abdominal VII casi recto. Pigóforo. Borde posteroventral proyectado en su tercio medio en una pequeña saliente con el tercio distal bifurcado y más estrecho que el tercio basal; lóbulos genitales elevados y redondeados (Fig. 17); parámeros medianos, delgados, con el ápice piloso, y rebasando el borde posterior del segmento abdominal VII (Fig. 18).

Medidas. Macho. Cabeza. Longitud total: 2.40; anchura a través de los ojos: 1.20; distancia interocular: 0.60; longitud total de los artejos antenales: I; 3.84; II, 1.20; III, 2.52; IV, no. Pronoto. Longitud total del lóbulo anterior: 1.08; longitud total del lóbulo posterior: 1.44; anchura máxima del lóbulo anterior: 1.80; anchura máxima del lóbulo posterior: 3.12. Longitud total del cuerpo: 10.8 . Hembra. No disponible.

\section{Resumen taxonómico}

Holotipo: macho. MÉXICO. SONORA. Rayón, 2975’N11040’O, 5-XI-1986, E. Mariño (IBUNAM).

Etimología. Nombre relacionado con la localidad tipo.

\section{Comentarios taxonómicos}

Los ángulos humerales del pronoto con la espina negra lo acerca a P. zebra, pero en esta última el borde posteroventral del pigóforo se proyecta en su tercio medio en una saliente mediana o larga (Fig. 7) y el lóbulo anterior del pronoto exhibe un patrón de coloración que combina franjas negras sobre un fondo amarillo y en $P$. rayonensis, el lóbulo anterior es anaranjado brillante, con el margen anterior y los ángulos frontales amarillos y el borde posteroventral posee una saliente muy pequeña (Fig.17). Los parámeros difieren como se aprecia en las figuras 8, 18.

Pselliopus mirabilis n. sp. (Figs. 19, 20, 33, 39, 46)

Holotipo macho. Coloración dorsal. Cabeza. Negra con las siguientes áreas de color amarillo: los juga, una mancha triangular entre los tubérculos anteníferos, una ancha franja transversal algo oblicua que corre del tercio anterior de los ojos hacia el centro pero sin llegar a tocarse, una mancha interocelar, una mancha algo cuadrada y pequeña cercana al borde posterior de los ojos y una pequeña mancha postocular. Pronoto. Lóbulo anterior negro con el margen anterior, los ángulos frontales, una mancha irregular a 
cada lado de la línea media y los bordes laterales de color amarillo; lóbulo posterior negro con las siguientes áreas de color amarillo: parte mesial de los bordes anterolaterales, una mancha casi circular a cada lado de la línea media, los márgenes posterolaterales (excepto la parte mesial que es negra), los procesos triangulares y el margen posterior. Hemélitro. Clavus y corium negros con reflejos pardo castaño. Abdomen. Segmentos dorsales II a VI negros con la parte media de los bordes laterales amarilla y el VII negro y con el margen posterior amarillo. Coloración ventral. Cabeza. Amarilla con la parte basal del rostro, un punto entre éste y cada ojo y una franja que corre de la región postocular al cuello de color negro. Tórax. Protórax amarillo con el prosterno y una franja ensanchada mesial que corre entre los acetábulos y el margen posterior de la propleura de color negro; mesotórax y metatórax negros, con el margen posterior amarillo y densamente pilosos. Patas. Coxas amarillas con 2 a 3 manchas negras en el tercio basal; fémures amarillos con 5 anillos negros; tibia anterior amarilla y con 4 anillos y la espina basal negros; tibia media amarilla y con 4 anillos negros; tibia posterior amarilla con 3 anillos y una franja que se alarga hacia el tercio distal negros; ápice de cada tibia negra. Abdomen. Esternito abdominal II amarillo delimitado por expansiones negras, III a V con 3 manchas subcuadradas amarillas delimitadas por expansiones negras y VI y VII amarillos con el margen posterior negro; margen posterior de cada esternito lateral y cercano al margen pleural ensanchado dando el aspecto de un círculo irregular negro; pigóforo amarillo con 2 franjas negras ensanchadas laterales a la línea media que se unen en el borde posteroventral; parámeros negros. Estructura. Pronoto. Ángulos humerales proyectados en una espina mediana, robusta y dirigida hacia fuera (Fig. 39). Abdomen. Borde posterior del segmento VII medianamente convexo. Pigóforo. Borde posteroventral proyectado en su tercio medio en una saliente mediana, cuyo tercio distal está bifurcado y es más estrecho que el tercio basal; lóbulos genitales elevados y redondeados (Fig. 19); parámeros cortos en forma de huso, relativamente delgados, con el ápice densamente piloso, y no tocan el borde posterior del segmento abdominal VII (Fig. 20).

Hembra. Coloración semejante al holotipo macho. Placas genitales amarillas con bandas oblicuas de color negro en el tercio apical.

Variación. Anillos negros del artejo antenal I ensanchados. Tonalidades amarillas del cuerpo más opacas.

Medidas. Primero el macho después la hembra. Cabeza. Longitud total: 2.16, 1.92; anchura a través de los ojos: 1.20, 1.08; distancia interocular: 0.60, 0.60; longitud total de los artejos antenales: I, 3.60, 3.84;II, 1.56, 1.56; III, 2.64, 2.64; IV, 1.68, no. Pronoto. Longitud total del lóbulo anterior: 1.08, 0.84; longitud total del lóbulo posterior: 1.68, 1.44; anchura máxima del lóbulo anterior: 1.68, 1.68; anchura máxima del lóbulo posterior: 3.12, 2.76. Longitud total del cuerpo: 9.96, 10.20.

\section{Resumen taxonómico}

Holotipo: macho. MÉXICO. NAYARIT, Mesa del Nayar, $22^{\circ} 21^{\prime} \mathrm{N}-104^{\circ} 65^{\prime} \mathrm{O}, 19-\mathrm{X}-1989$, A. Cadena (IBUNAM). Paratipos. MÉXICO. NAYARIT. 2 machos, Mesa del Nayar, $22^{\circ} 21^{\prime} \mathrm{N}-104^{\circ} 65^{\prime} \mathrm{O}, 19-\mathrm{X}-1989$, A. Cadena (IBUNAM).

Etimología. Nombre específico haciendo alusión al conspicuo desarrollo del pigóforo.

\section{Comentarios taxonómicos}

El pigóforo de $P$. majesticus (Fig. 11) se asemeja al de $P$. mirabilis, (Fig. 19), pero los parámeros son más largos (Figs. 12, 20) y la coloración del cuerpo particularmente del pronoto y de los esternitos abdominales muy distintos, destacando las tonalidades rojizas de P. majesticus y los tonos más pálidos en $P$. mirabilis. La coloración general del cuerpo y los parámeros reducidos (Figs. 20, 46) sin alcanzar a rebasar el borde posterior del segmento abdominal VII lo acercan a P. dantei, pero en este último la proyección media del borde posteroventral del pigóforo está extraordinariamente alargada (Fig. 3) y el borde posterior del segmento abdominal VII ampliamente arqueado (Fig. 29) en comparación con P. mirabilis cuya proyección posteroventral del pigóforo es más corta (Fig. 19) y el borde posterior del segmento abdominal VII medianamente convexo (Fig. 33). Los parámeros de ambas especies también difieren (Figs. 4, 20)

Pselliopus marmorosus n. sp. (Figs. 23, 24, 30, 40, 48)

Holotipo macho. Coloración dorsal. Cabeza. Negra con la región anterior de los juga, una pequeña mancha cercana al tercio medio de cada ojo y una mancha triangular interocelar amarilla. Pronoto. Lóbulo anterior negro, con los ángulos frontales y la mitad distal de los márgenes anterolaterales amarillos; lóbulo posterior negro con las siguientes áreas de color amarillo: los márgenes anterolaterales (excepto la región humeral negra), una mancha irregular anterior a cada lado de la línea media, los márgenes posterolaterales (excepto la parte mesial negra), los procesos triangulares y el tercio medio del borde posterior. Hemélitro. Clavus negro; corium pardo castaño pálido con las venas oscuras. Abdomen. Segmentos dorsales II a VI negros y con el tercio medio de los márgenes laterales amarillos, y el VII amarillo y con el margen anterior negro. Coloración ventral. Cabeza. Amarilla con la región situada entre la búcula y los ojos (excepto una mancha semicircular amarilla en la cara inferior de los tubérculos anteníferos) 
y la franja que corre de la parte posterior de cada ojo al cuello negra. Tórax. Protórax negro con el prosterno, gran parte del margen anterior de la propleura, una mancha oval en el margen superior de la propleura, el margen posterior del protorax y los acetábulos amarillos; mesotórax y metatórax negros, densamente pilosos y con la mitad posterior de los acetábulos amarillos. Patas. Coxas amarillas con la cara interna y el borde inferior pardo o negro; fémures amarillos con 5 anillos negros (el primero incompleto), y con un punto discoidal también negro en la cara anterior entre los anillos III y IV; tibia anterior amarilla con 3 anillos y la espina apical negras; tibia media amarilla y con 3 anillos negros; tibia anterior y media con una mancha difusa subapical pardo claro; tibia posterior amarilla con 3 anillos y una franja que se alarga hacia el tercio distal negra. Abdomen. Esternitos abdominales II a VI amarillos con el margen posterior negro, que lateral y cercana al margen pleural se ensancha, dando el aspecto de un círculo irregular negro; esternito VII amarillo con el tercio medio del borde posterior negro; pigóforo amarillo y con 2 manchas negras subcuadradas que se unen en el borde posteroventral; parámeros con el ápice negro y el resto amarillo. Estructura. Pronoto. Ángulos humerales sin espina y romos (Fig. 40). Abdomen. Borde posterior del segmento VII suavemente convexo (Fig. 30). Pigóforo. Borde posteroventral proyectado en su tercio medio en una saliente mediana, cuadrada, con la mitad distal igual de ancha que la mitad basal; lóbulos genitales planos, y no elevados (Fig. 23); parámeros delgados, largos, con el ápice piloso y rebasando el borde posterior del segmento abdominal VII (Fig. 24).

Hembra. Coloración semejante al holotipo macho. Placas genitales amarillas con bandas oblicuas de color negro en el tercio apical.

Variación. Lóbulo posterior del pronoto sin manchas amarillas a cada lado de la línea media.

Medidas. Primero el macho después la hembra. Cabeza. Longitud total: 2.64, 2.28; anchura a través de los ojos: 1.32, 1.20; distancia interocular: 0.72, 0.60; longitud total de los artejos antenales: I; 3.00, 3.00; II, 1.44, 1.44; III, 2.04, 2.04; IV, 1.32, 1.32. Pronoto. Longitud total del lóbulo anterior: 1.44, 1.44; longitud total del lóbulo posterior: 1.80, 1.68; anchura máxima del lóbulo anterior: 2.28, 2.04; anchura máxima del lóbulo posterior: 3.60, 3.48. Longitud total del cuerpo: 13.56, 12.60.

\section{Resumen taxonómico}

Holotipo: macho. MÉXICO. SINALOA. 5 mi. al O de El Palmito 6000', 235'ㅅ-10583'O, 13-X-1975, J. Powell, J. Chemsak, T. Eichlin y T. Friedlander (UCB). Paratipos: MÉXICO. SINALOA. 1 hembra, El Palmito 6 mi. al O, 24-IX-1977, J. Chemsak y A. y M. Michelbacher (UCB); 1 hembra, 8 mi. al O de El Palmito. 6000', 12-X-
1975, J. Powell, J. Chemsak, T. Eichlin y T. Friedlander (IBUNAM). ESTADOS UNIDOS DE AMÉRICA. ARIZONA. 1 macho, 1 hembra, Cochise Co. Chiricahua Mts., 2 Mi SO Portal Sunny Flat. Campground., 26-VII1977, R.E. y P.L. Coville (UCB); 1 macho, Cochise Co. Rucker Canyon Forest Campground Chiricahua Mts., 21IX-70, P.S. Bartholomew (UCB).

Etimología. Nombre específico haciendo referencia a la coloración del pronoto; del latín, marmorosus, como mármol.

\section{Comentarios taxonómicos}

Especie inconfundible por tener los ángulos humerales del lóbulo posterior del pronoto romos, sin una espina o tubérculo, como también ocurre en P. inermis (Fig. 40). En P. marmorosus, la mayor parte del lóbulo posterior del pronoto, del mesotórax y del metatórax son negros y el tercio medio del borde posteroventral del pigóforo está proyectado en una saliente mediana, cuadrada, e igual de ancha en la base que en el ápice (Fig. 23). En P. inermis el lóbulo posterior del pronoto, el mesotórax y el metatórax son mayormente de color amarillo o anaranjado castaño pálido y la proyección media del borde posteroventral del pigóforo es muy pequeña y algo aguda (Fig. 21). Los parámeros de ambas especies son parecidos (Figs. 22, 24).

Pselliopus promeceops n. sp. (Figs. 5, 6, 34, 41, 47)

Holotipo macho. Coloración dorsal. Cabeza. Negra, con la parte anterior de los juga, una mancha triangular entre los tubérculos anteníferos, la zona adyacente a los ojos y una mancha interocelar, de color amarillo. Pronoto. Lóbulo anterior negro con el margen anterior, los ángulos frontales, una mancha irregular a cada lado del tercio medio y gran parte de los márgenes anterolaterales de color amarillo; lóbulo posterior amarillo con las siguientes áreas de color negro: margen anterior anexo al lóbulo anterior, una franja longitudinal media que se extiende hasta cerca del margen posterior, gran parte de los márgenes posterolaterales y del margen posterior, la espina humeral y zonas adyacentes a ella, y la parte mesial de los márgenes posterolaterales. Hemélitro. Clavus y endocorium pardo castaño y el resto del corium anaranjado palido. Abdomen. Segmentos dorsales del abdomen II a VI amarillos, con la parte mesial pardo oscura y con una franja transversal negra cercana al margen anterior; segmento VII amarillo. Coloración ventral. Cabeza. Amarilla, con una franja que corre de la parte anterior de cada ojo a la base del rostro, los márgenes laterales del cuello y una proyección orientada hacia los ojos negros. Tórax. Protórax amarillo, con una franja ensanchada de color negro corriendo en el tercio medio de la propleura por encima de los acetábulos hasta 
alcanzar el tercio medio de la propia propleura; borde posterior del protórax pardo oscuro; mesotórax y metatórax negros, densamente pilosos, con la mitad posterior de los acetábulos y el margen posterior del mesotórax amarillos. Patas. Coxas amarillas con una mancha irregular negra en la cara anterior y en el borde externo; fémures amarillos, los anteriores con 5 anillos negros, el primero y el tercero incompletos y con puntos también negros entre los anillos 2 y 3, los fémures medios con 4 anillos negros y un punto también negro entre los anillos 1 y 2 y los fémures posteriores con 6 anillos, de ellos el 1 y 3 incompletos; tibias amarillas con 3 anillos negros en la mitad basal y con el ápice pardo oscuro; tibia posterior con una mancha subapical alargada pardo claro. Abdomen. Esternitos abdominales II a VI amarillos con una franja transversal intersegmental negra, que lateralmente y cercana al margen pleural se ensancha, dando el aspecto de un círculo irregular negro; pigóforo amarillo, con el borde caudal y 2 franjas ensanchadas sobre los márgenes laterales negros; parámeros amarillos y con el tercio distal negro. Estructura. Pronoto. Ángulos humerales proyectados en una espina larga, fina y dirigida hacia fuera (Fig. 41). Abdomen. Borde posterior del segmento VII recto (Fig. 34). Pigóforo. Borde posteroventral proyectado en su tercio medio en una saliente pequeña y roma con el tercio distal más estrecho que el basal y ligeramente bifurcado; lóbulos genitales elevados y redondeados (Fig. 5); parámeros delgados, medianos, dirigidos caudalmente hacia fuera, con el ápice piloso, y en una vista imaginaria rebasan el borde posterior del segmento abdominal VII. (Fig. 6).

Hembra. Coloración semejante al holotipo macho. Placas genitales amarillas con bandas oblicuas de color negro en el tercio apical.

Variación. Margen anterior del pronoto amarillo o con una mancha pardo oscura cercana a los ángulos frontales. Corium enteramente pardo oscuro. Pigóforo amarillo con los márgenes caudal y lateral uniformemente pardo oscuro.

Medidas. Primero el macho después la hembra. Cabeza. Longitud total: 2.04, 2.04; anchura a través de los ojos: 1.08, 0.96; distancia interocular: $0.60,0.60$; longitud total de los artejos antenales: I; 3.72, 3.72; II, 1.32, 1.20; III, 2.28, 2.40; IV, no, 1.92. Pronoto. Longitud total del lóbulo anterior: $0.84,0.84$; longitud total del lóbulo posterior: 1.32, 1.32; anchura máxima del lóbulo anterior: 1.44, 1.44; anchura máxima del lóbulo posterior: 2.76, 2.64. Longitud total del cuerpo: 9.84, 9.60.

\section{Resumen taxonómico}

Holotipo: macho. MÉXICO. JALISCO. Km. 29 carr. El Tuito-Tehualmixtle, $20^{\circ} 22^{\prime} \mathrm{N}-105^{\circ} 54^{\prime} \mathrm{O}, 24-\mathrm{XI}-1991$. E. Ramírez y J. Villa. (IBUNAM). Paratipos. MÉXICO. GUERRERO. 1 macho, Acahuizótla, 27-VI-1983, M.
García (IBUNAM). MICHOACÁN. 1 macho, 1 hembra, km 92 carr. Pátzcuaro-Nueva Italia, $19^{\circ} 15^{\prime} \mathrm{N}-102^{\circ} 03^{\prime} \mathrm{O}$, 13-VII-1983. E Barrera (IBUNAM); 1 hembra, Charanda, 22-IX-1987. E. Barrera, R. Barba y D. Barrera (IBUNAM); JALISCO. 1 hembra, Sierra del Cuale, $20^{\circ} 73^{\prime} \mathrm{N}-103^{\circ} 48^{\prime} \mathrm{O}$, 18-IX-1988, E. Ramírez (IBUNAM); 3 hembras, km 159 carr. Pto. Vallarta-Manzanillo, $19^{\circ} 66^{\prime} \mathrm{N}-105^{\circ} 18^{\prime} \mathrm{O}, 25-\mathrm{IX}-$ 1990, E. González, G. Ortega, C. Mayorga y S. Rodríguez (IBUNAM); NAYARIT. 1 hembra, Jalcocotán, 2147'N10509’O, 30-VII-1984, M García (IBUNAM); 1 hembra, km 15 carr., Mecatán-Sta. Cruz, 21 ²5’N-104³2’O, 30VII-1984, E. Barrera (IBUNAM).

Etimología. Del griego, promeces, alargado y ops, cara.

\section{Comentarios taxonómicos}

Especie cercana a $P$. nigropictus compartiendo las largas espinas negras de los ángulos humerales del pronoto (Figs. 36, 41), el desarrollo de la proyección mesial del borde posteroventral del pigóforo que es pequeña y roma (Figs. 5,27 ), y el tener el borde posterior del segmento abdominal VII prácticamente recto y no arqueado (Figs. 34, 35). El parámero de $P$. promeceops, es más largo, esbelto y dirigido caudalmente (Fig. 6) y el lóbulo anterior del pronoto es negro y con manchas amarillas aisladas, mientras que en $P$. nigropictus los parámeros son muy pequeños y ensanchados (Fig. 28) y el lóbulo anterior tiene numerosas franjas longitudinales negras sobre un fondo amarillo.

\section{Pselliopus karlenae Hussey \\ Pselliopus karlenae Hussey 1954: 153-156.}

Los datos distribucionales de esta especie estaban limitados a los que da la descripción original donde se cita para Honduras (Departamento Morazán, Mt. Caculatepe).Cinco machos y 3 hembras recolectados en Chiapas, señalan el primer registro de esta especie para México.

\section{Resumen taxonómico}

MÉXICO. CHIAPAS. 2 machos, 2 hembras, Municipio Zinacantan, Paraje Vobits, 1371 m, 18-VIII-1976, D. E. Y J. A. Breedlove (CAS).; 1 macho, 26 km E Comitán, 4VIII-1991, C. Mayorga y R. Jones (IBUNAM); 2 machos, km 141 carr., Comitán-San Cristóbal de las Casas, 21VII-1978, G. Ortega-León (IBUNAM); 1 hembra, Laguna Chamula, nr. Cruz Quemada, en hwy 190, entre Comitán y Teopisca, 2438 m, 18-X-1974, D. E. Y J. A. Breedlove (CAS).

\section{Agradecimientos}

A los siguientes colegas e instituciones por haber facilitado en préstamo parte del material aquí estudiado: 
Mick Webb (BMNH), John Rawlins y Robert Davidson (CMNH), y Vincent Lee y Keve Ribardo (CAS). A Luis García Prieto (UNAM) por el apoyo en computación. De manera especial a la Dirección General de Asuntos del
Personal Académico (DGAPA) por el apoyo financiero al proyecto "Análisis comparativo de la coreidofauna en tres estados de la República Mexicana: Guerrero, Oaxaca y Chiapas (Hemiptera-Heteroptera)" (IN223503-3).

\section{Clave para separar las especies mexicanas de Pselliopus (machos) *}

1. Fémures con anillos negros y abundantes puntos también negros entre éstos . . .2

1'. Fémures sólo anillados de negro y eventualmente con un punto negro subdistal

2. Lóbulo anterior del pronoto provisto de ocho largas y agudas espinas

2'. Lóbulo anterior del pronoto tuberculado o con 16 ó más espinas medianas o cortas.

3.

Lóbulo anterior del pronoto tuberculado.

..P. tuberculatus (Champion)

3 .

Lóbulo anterior del pronoto adornado con 16 ó más espina. P. spinicollis (Champion)

4. Parámeros apicalmente clavados (Fig. 9, 10).

5. Borde posteroventral del pigóforo proyectado en su tercio medio en una saliente muy pequeña y delgada (Fig. 15)... .P. mexicanus (Champion)

5. Borde posteroventral del pigóforo proyectado en su tercio medio en una saliente mediana, y conspicuamente ensanchada hacia su base (Fig. 9). P. cosmopolites n. sp.

6. Borde posteroventral del pigóforo proyectado en su tercio medio en una saliente muy pequeña y delgada o algo robusta (Figs. 1, 5, 17, 21, 27).

6'. Borde posteroventral del pigóforo proyectado en su tercio medio en una saliente mediana o muy larga (Figs. 3 ,

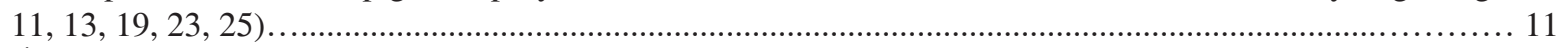

7. Ángulos humerales del pronoto romos, no espinados o a lo sumo con un diminuto nódulo (Fig. 43).......

P. inermis (Champion)

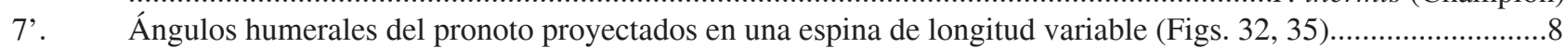

8. Ángulos humerales del pronoto proyectados en una espina corta y robusta....................................................

8'. Ángulos humerales del pronoto proyectados en una espina larga y robusta (Fig. 32)....................................10

9. Parámeros conspicuamente alargados y rebasando el borde posterior del segmento abdominal VII (Fig. 2); lóbulo anterior del pronoto mayormente negro................................................. ivanicus Brailovsky y Barrera

9'. Parámeros medianos (Fig. 18 ); lóbulo anterior del pronoto anaranjado brillante y con el margen anterior y los ángulos frontales amarillos.

P. rayonensis $\mathrm{n}$. $\mathrm{sp}$.

10. Parámero pequeño y ensanchado (Fig. 28); lóbulo anterior del pronoto con numerosas franjas longitudinales negras sobre un fondo amarillo...................................................................... nigropictus (Champion)

10'. Parámero mas largo, esbelto y dirigido caudalmente (Fig. 6); lóbulo anterior del pronoto negro y con manchas amarillas aisladas.......................................................................................... promeceops $\mathrm{n} . \mathrm{sp}$.

11. Tercio medio del borde posteroventral del pigóforo proyectado en una saliente cuadrada (Fig. 23) ................12

11'. Tercio medio del borde posteroventral del pigóforo proyectado en una saliente en forma de lanza o bien larga y aguda......

12. Lóbulo anterior del pronoto con un tubérculo bien definido a cada lado de la línea media; ángulos humerales del pronoto proyectados en una espina corta y robusta; dorso cefálico y el lóbulo anterior del pronoto mayormente anaranjado amarillento. P. flaviceps Brailovsky y Barrera

12'. Lóbulo anterior del pronoto desprovisto de un tubérculo a los lados de la línea media; ángulos humerales del pronoto romos, no espinados (Fig. 40); lóbulo anterior del pronoto negro y con los ángulos frontales amarillos; dorso cefálico negro y con una mancha amarilla interocelar.

P. marmorosus n. sp.

13. Lóbulo anterior y posterior del pronoto prácticamente todo el de color anaranjado brillante .....

P. latispina Hussey

13'. Lóbulo anterior del pronoto amarillo o negro y el posterior anaranjado, o rojizo, o negro y nunca unicoloros 
14. Borde posteroventral del pigóforo proyectado en su tercio medio en una saliente mediana en forma de triángulo equilátero, basalmente ensanchado y que no alcanza a tocar el borde posterior del segmento abdominal VII (Fig. 25) .P. ventus Brailovsky y Barrera

14'. Borde posteroventral del pigóforo proyectado en su tercio medio en una larga saliente en forma de triángulo isósceles que alcanza o rebasa el borde posterior del segmento abdominal VII (Fig. 13).

15. Espinas de los ángulos humerales del pronoto roja (Fig. 14).................................... P. rufofasciatus (Champion)

15'. Espinas de los ángulos humerales del pronoto negras.

16. Proyección media del borde posteroventral del pigóforo conspicuamente alargada (Fig. 3); borde posterior del segmento abdominal VII extraordinariamente arqueado (Fig. 29); parámeros pequeños, no alcanzando el tercio medio de la proyección mesial del pigóforo (Fig. 4) . P. dantei Brailovsky y Barrera

16'. Proyección media del borde posteroventral del pigóforo alargada; borde posterior del segmento abdominal VII no conspicuamente arqueado (Figs. 7, 11, 19); parámeros medianos, alcanzando o rebasando el tercio medio de la proyección medial del pigóforo (Figs. 8,12, 2).

17. Parámero mediano, esbelto, alcanzando el tercio medio de la proyección media del pigóforo (Fig. 20) ...........18

17'. Parámero alargado, robusto, rebasando el tercio medio de la proyección media del pigóforo $($ Fig. 8, 42) ............

18. Proyección media del borde posteroventral de pigóforo esbelto (Fig. 20); borde posterior del segmento abdominal VII algo convexo (Fig. 33); parámero muy corto (Fig. 20)...

P. mirabilis n. $\mathrm{sp}$.

18'. Proyección media del borde posteroventral del pigóforo robusta y ensanchada (Fig. 11); borde posterior del segmento abdominal VII arqueado; parámero más largo (Fig. 12).

19. Lóbulos genitales elevados (Fig. 11); parámero recto (Fig. 12); proyección media del borde posteroventral del pigóforo nunca en forma de lanza (Fig. 11) .............................................. P. majesticus Brailovsky y Barrera

19'. Lóbulos genitales poco elevados; parámero curvo; proyección media del borde posteroventral del pigóforo en forma de lanza P. karlenae Hussey

*De P. lineaticeps sólo se conocen dos hembras.

\section{Literatura citada}

Barber, H. G.1924. A new species of Pselliopus (Hemiptera: Reduviidae). Proceedings of the Entomological Society of Washington 26: 211-213.

Brailovsky, H. y E. Barrera. 2004. Especies nuevas de Pselliopus (Hemiptera: Heteroptera: Reduviidae: Harpactorinae) de México. Anales del Instituto de Biología, Universidad Nacional Autónoma de México, Serie Zoología 75: 313-330.

Champion, G. C. 1899. Biologia Centrali-Americana. Insecta. Rhynchota. Hemiptera-Heteroptera vol. II. In Goodwin and Salvin (eds.), London. p. 244-250 + Lámina 15.

Coscarón, M. C. 2002. Reduviidae. In: Llorente Bousquets J. y J. J. Morrone (eds.) Biodiversidad, taxonomía y biogeografía de artrópodos de México. Hacia una síntesis de su conocimiento, vol. 3. Museo de Zoología, Facultad de Ciencias, Universidad Nacional Autónoma de México, México. D.F. p. 455-466.

Fracker, S. B. 1912. A systematic outline of the Reduviidae of North America. Proceedings of the Iowa Academy of Science 19: 217-247.

Hussey, R. F. 1954. Two new species of Pselliopus and some distributional notes (Hemiptera, Reduviidae). The Pan-Pacific Entomologist 30: 153-158.

Maldonado Capriles, J. 1990. Systematic catalogue of the Reduviidae of the World (Insecta: Heteroptera). Caribbean Journal of Science. Special Edition: 694 p.

Schuh, R. T. y J. A. Slater. 1995. True bugs of the World (Hemiptera: Heteroptera). Classification and natural history. Cornell University Press, Ithaca and London $350 \mathrm{p}$.

Stål, C. 1862. Hemiptera Mexicana enumeravit speciesque novas descripsit. Stettin. Entomologische-Zeitung: 448449.

Stål, C. 1872. Enumeratio Hemipterorum. Kongliga Svenska Vetenskaps Akademiens Handlingar 10 (4): 86-87. 

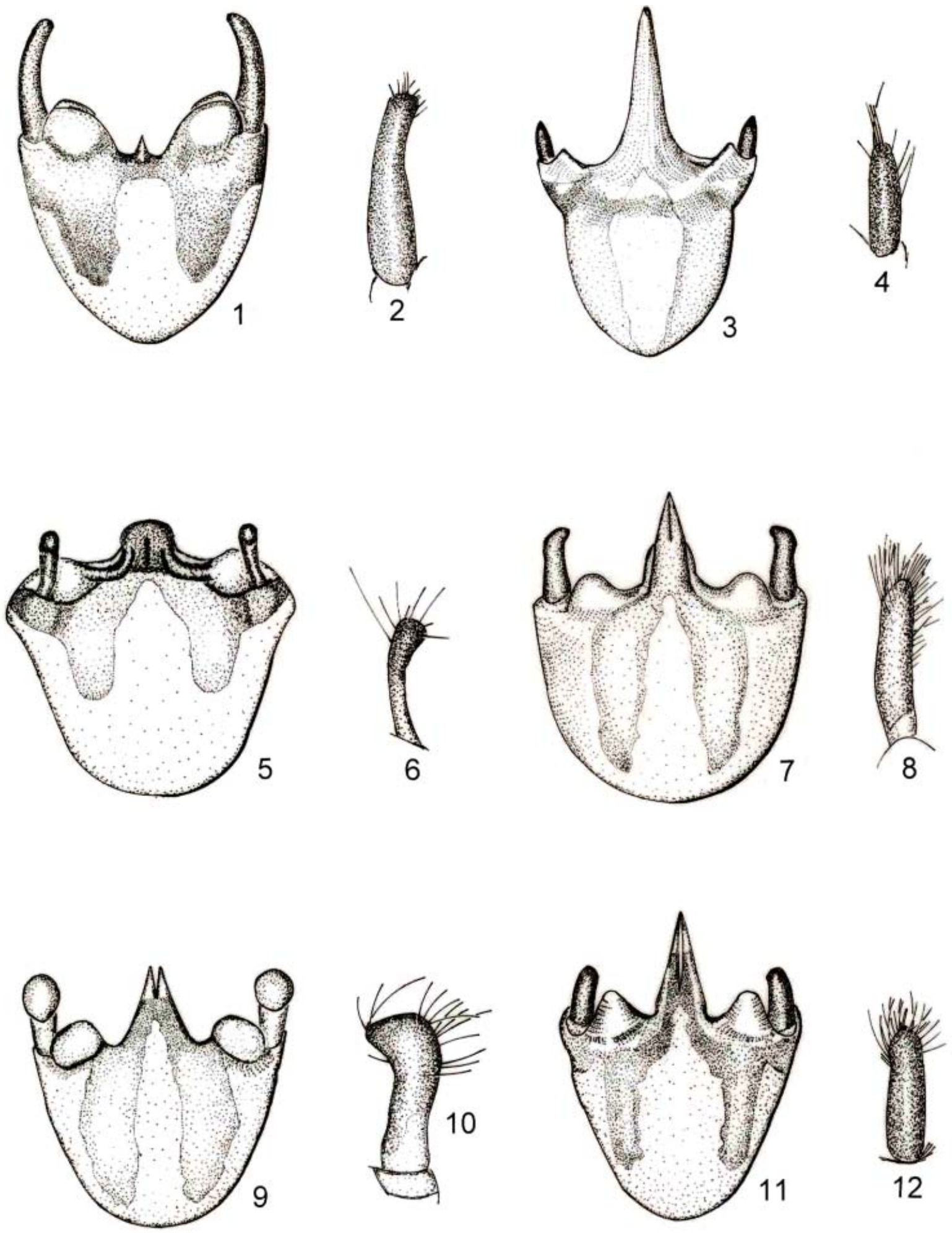

Figuras 1-12. Pselliopus spp. 1-2. P. ivanicus Brailovsky y Barrera. 1. Pigóforo en vista caudal. 2. Parámero. 3-4. P. dantei Brailovsky y Barrera. 3. Pigóforo en vista caudal. 4. Parámero. 5-6. P. promeceops n. sp. 5. Pigóforo en vista caudal. 6. Parámero. 7-8. P. zebra (Stål). 7. Pigóforo en vista caudal. 8. Parámero. 9-10. P. cosmopolites n.sp. 9. Pigóforo en vista caudal. 10. Parámero. 11-12. P. majesticus Brailovsky y Barrera. 11. Pigóforo en vista caudal. 12. Parámero. 

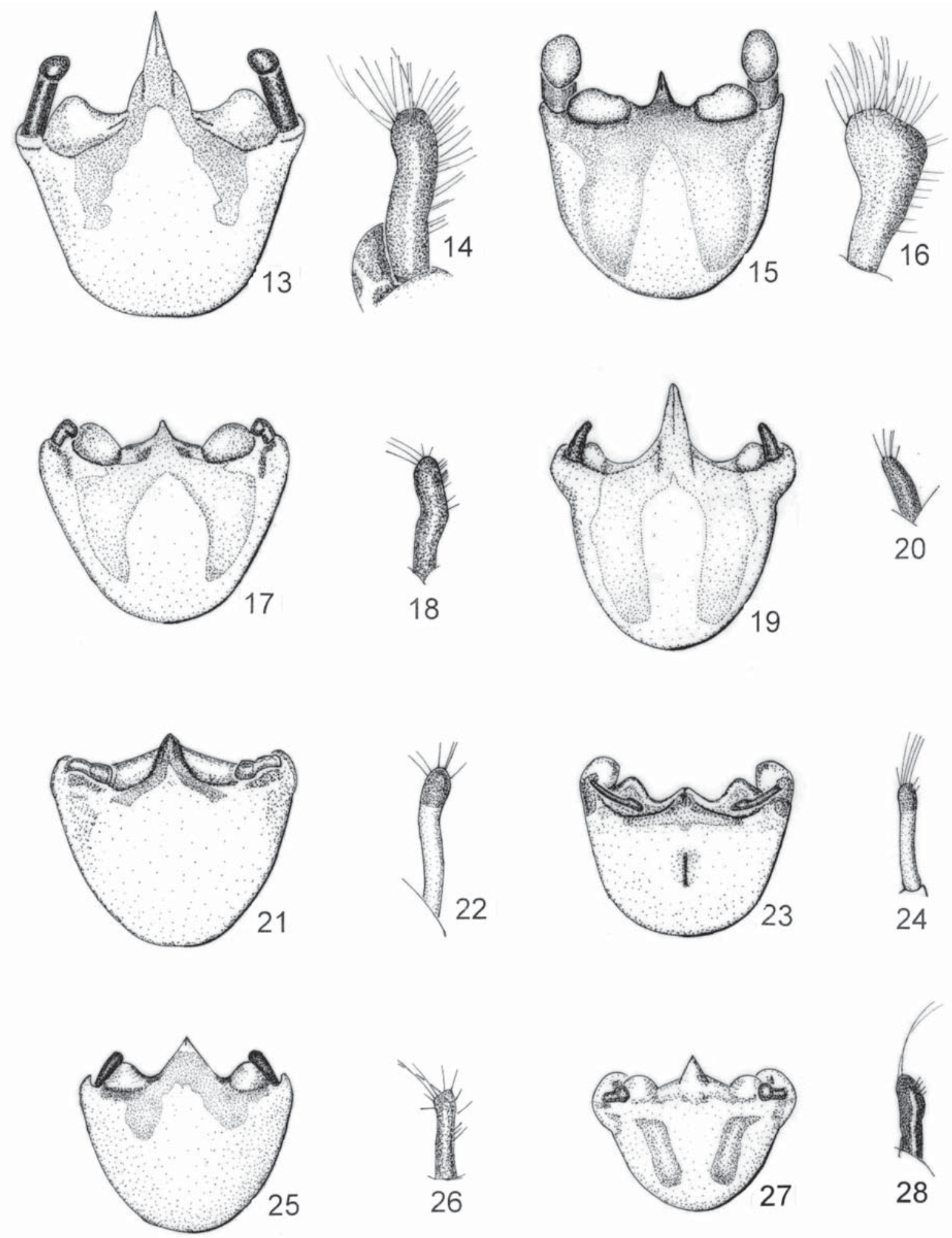

Figuras 13-28. Pselliopus spp. 13-14. P. rufofasciatus (Champion). 13. Pigóforo en vista caudal. 14. Parámero. 15-16. P. mexicanus (Champion). 15. Pigóforo en vista caudal. 16. Parámero. 17-18. P. rayonensis n. sp. 17. Pigóforo en vista caudal. 18. Parámero. 19-20. P. mirabilis n. sp. 19. Pigóforo en vista caudal. 20. Parámero. 21-22. P. inermis (Champion). 21. Pigóforo en vista caudal. 22. Parámero. 23-24. P. marmorosus n. sp. 23. Pigóforo en vista caudal. 24. Parámero. 25-26. P. ventus Brailovsky y Barrera. 25. Pigóforo en vista caudal. 26. Parámero. 27-28. P. nigropictus (Champion). 27. Pigóforo en vista caudal. 28. Parámero. 

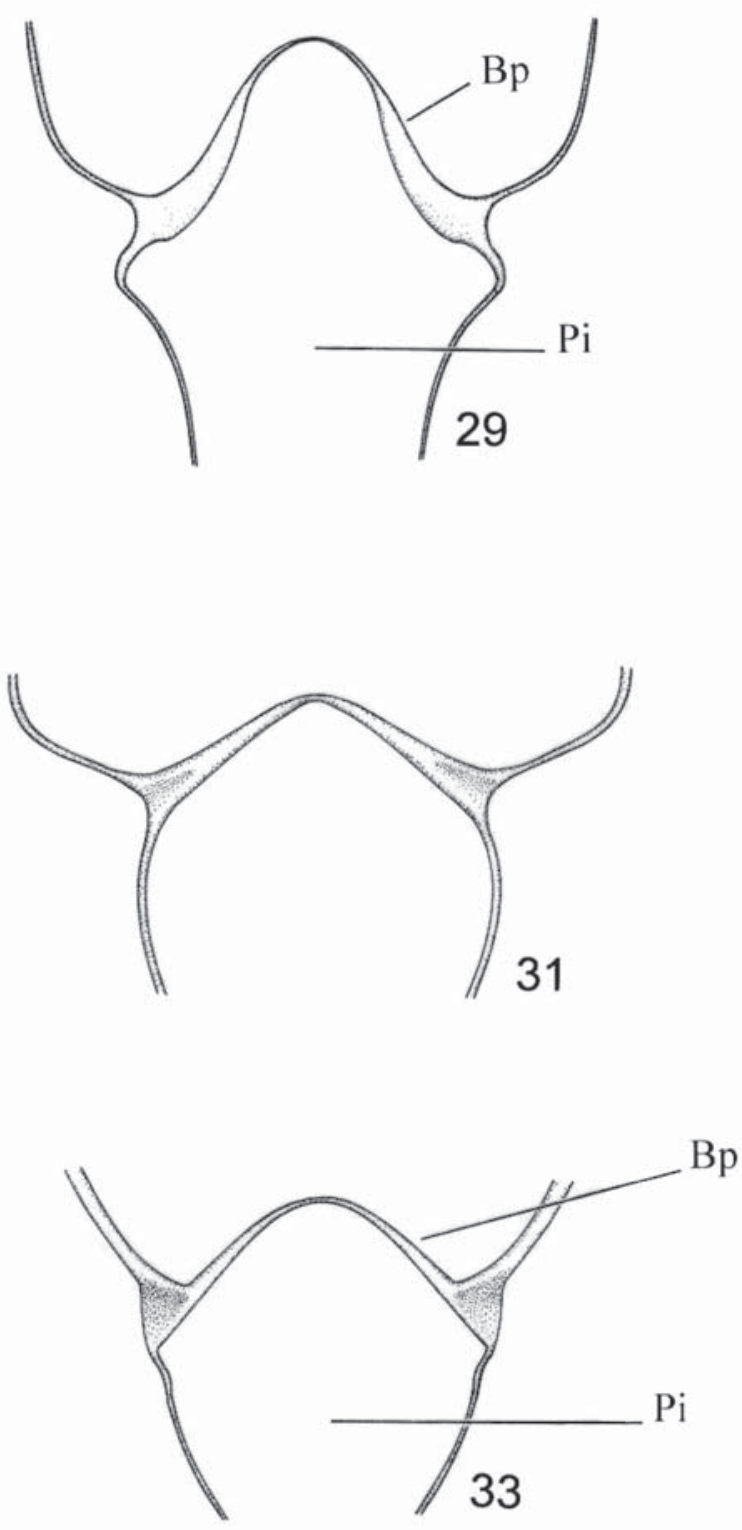
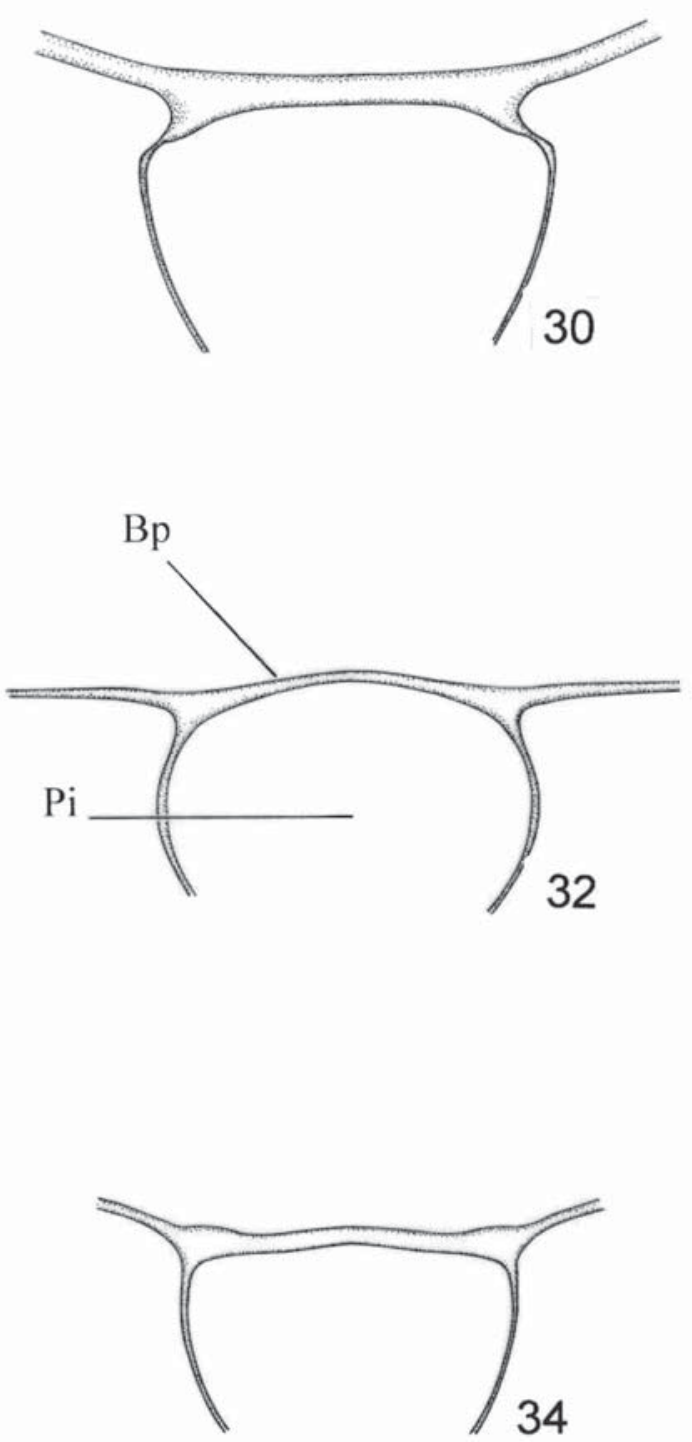

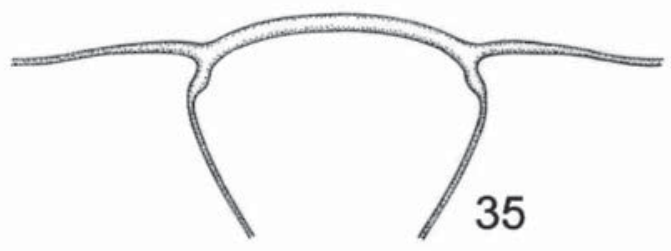

Figuras 29-35. Pselliopus spp. Vista caudal del borde posterior del segmento abdominal VII (macho). 29. P. dantei Brailovsky y Barrera. 30. P. marmorosus n. sp. 31. P. cosmopolites n. sp. 32. P. rayonensis n. sp. 33. P. mirabilis n. sp. 34. P. promeceops $\mathrm{n}$. sp. 35. P. nigropictus (Champion). $\mathrm{Bp}=$ borde posterior del segmento abdominal VII del macho; pi= pigóforo en vista caudal. 


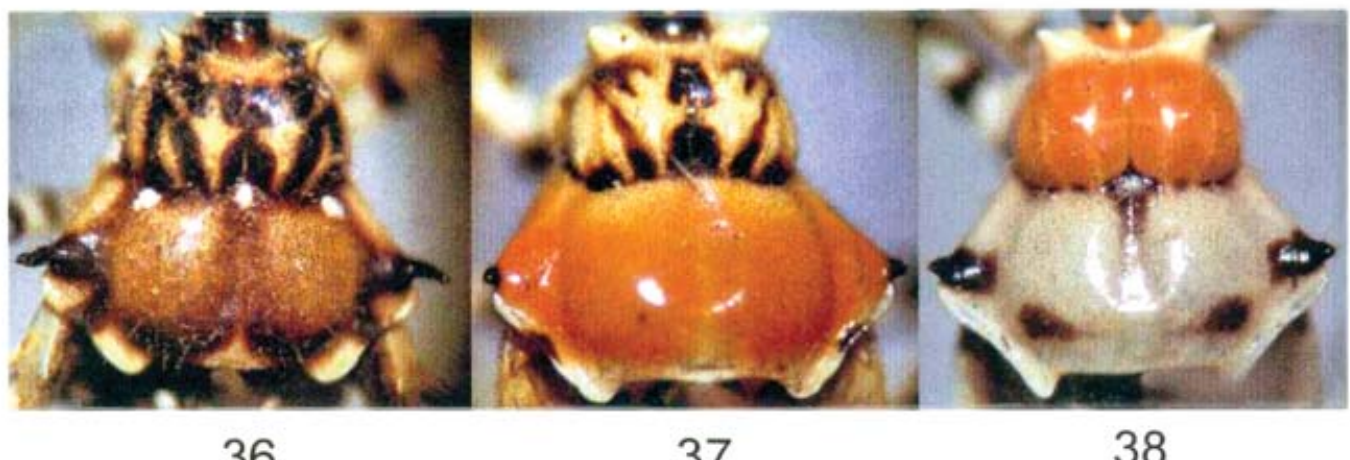

36

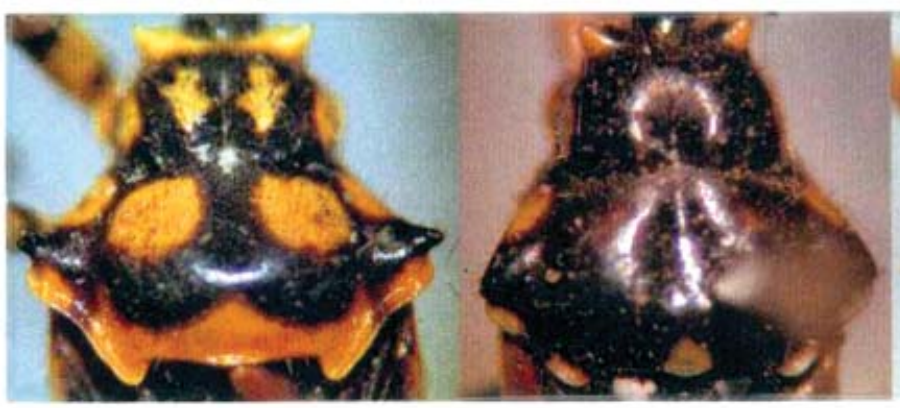

39
40

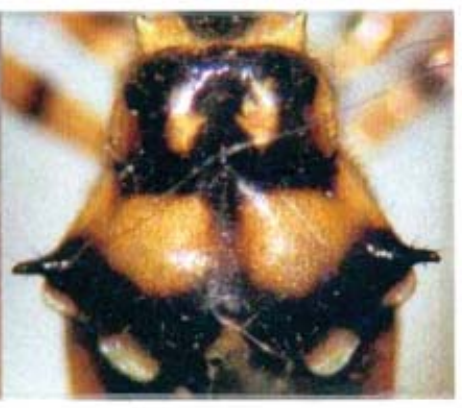

41

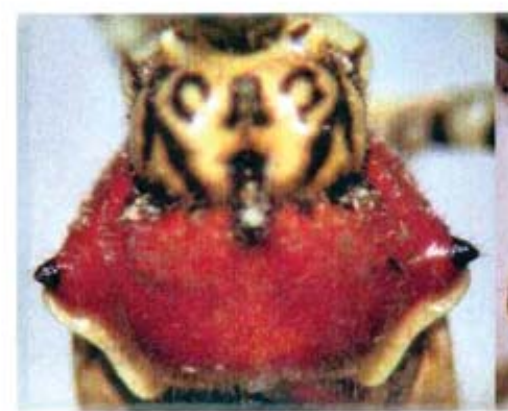

42

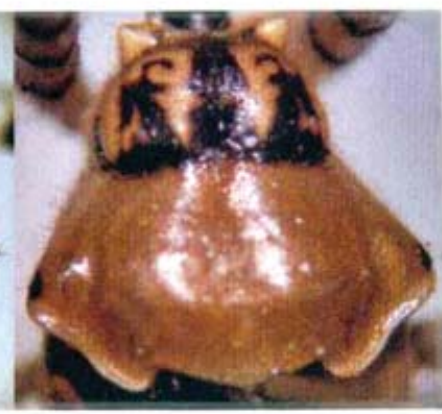

43

Figuras 36-43. Pselliopus spp. Pronoto. 36. P. nigropictus (Champion). 37. P. cosmopolites n. sp. 38. P. rayonensis n. sp. 39. P. mirabilis n.sp. 40. P. marmorosus n. sp. 41. . promeceops n. sp. 42. P. zebra (Stål). 43. P. inermis (Champion). 


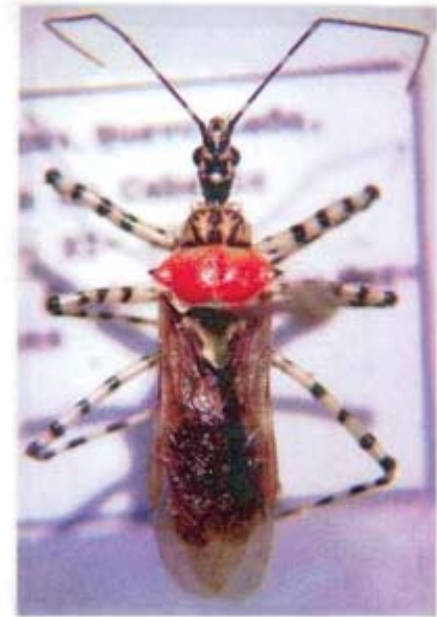

44

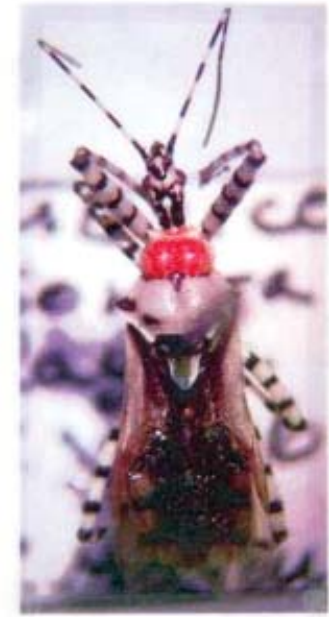

45

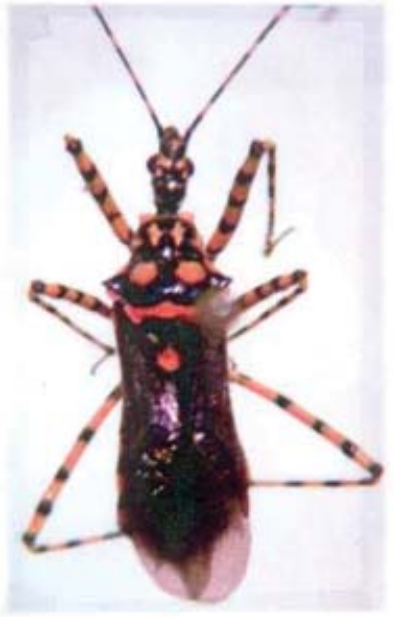

46

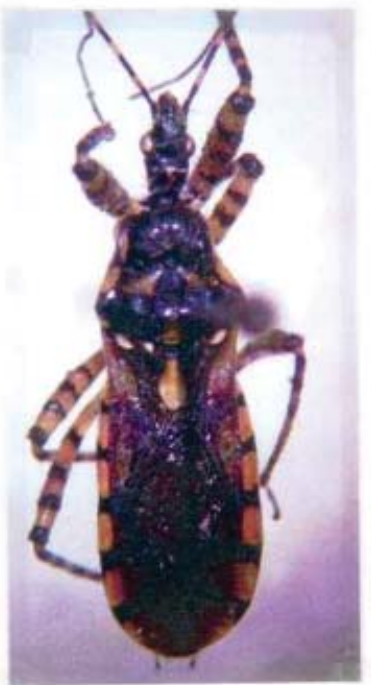

47

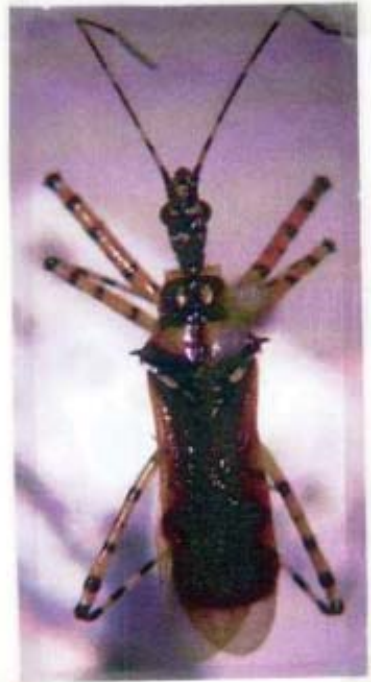

48

Figuras 44-48. Vista dorsal de Pselliopus spp. 44. P. cosmopolites n.sp. 45. P. rayonensis n. sp. 46. P. mirabilis n. sp. 47. P. promeceops n. sp. 48. P. marmorosus n. sp. 